\title{
М.В. Самойленко
}

\section{СТОХАСТИЧЕСКИЙ МАТРИЧНО-ИТЕРАЦИОННЫЙ МЕТОД РЕШЕНИЯ СИСТЕМ ЛИНЕЙНЫХ АЛГЕБРАИЧЕСКИХ УРАВНЕНИЙ И ЕГО ПРИМЕНЕНИЕ В ОБРАБОТКЕ ИЗОБРАЖЕНИЙ}

\begin{abstract}
Самойленко Марина Витальевна, кандидат технических наук, доцент, окончила Московский авиационный институт и Московский физико-технический институт. Доцент Московского авиационного института (национального исследовательского университета). Имеет монографии, статьи, изобретения. Область научных интересов - обработка сигналов и изображений. [e-mail: Samoi.Mar@mail.ru].
\end{abstract}

Аннотация

В работе предложен новый метод решения систем линейных алгебраических уравнений (СЛАУ) - стохастический матрично-итерационный метод. Этот метод разработан для широкого класса задач, приводящихся к недоопределенным СЛАУ, в которых присутствуют ошибки измерений. При представлении СЛАУ векторно-матричным уравнением искомые переменные объединяются в вектор-оригинал. Применимость метода ограничена характером восстанавливаемого вектора-оригинала: основная часть его компонент должна быть равна известному фоновому значению, а ограниченное количество компонент - превышать это значение. При обработке изображений это условие означает, что речь идет об ограниченном числе ярких объектов на постоянном фоне.

Применение предложенного метода продемонстрировано на примерах восстановления размытых и зашумленных изображений. Задача приводится к СЛАУ, в которой вектор-отображение представляет собой строчно-столбцовую развертку искаженного изображения, а восстанавливаемый вектор-оригинал - строчно-столбцовую развертку исходного неискаженного изображения. Проведено компьютерное моделирование в среде MATLAB, которое позволило определить оптимальные параметры стохастического матрично-итерационного метода и продемонстрировать его преимущества в условиях аддитивных шумов.

Ключевые слова: стохастический матрично-итерационный метод, матрично-итерационный метод, СЛАУ, аддитивный шум, восстановление изображения, функция рассеяния точки.

doi:

\section{STOCHASTIC MATRIX-ITERATIVE METHOD AND ITS APPLICATION IN THE IMAGES PROCESSING}

Marina Vitalevna Samoilenko, Candidate of Science in Engineering, Associate Professor; graduated from Moscow Aviation Institute and the Moscow Institute of Physics and Engineering; Associate Professor at Moscow Aviation Institute (National Research University); an author of articles, monographs, and inventions in the field of signal and image processing.e-mail:Samoi.Mar@mail.ru.

\section{Abstract}

The article deals with a new method for solving of the systems of linear algebraic equations (SLAE). It is a stochastic matrix-iterative method. This method is designed for a wide class of problems in which measurement errors are present. When the SLAE is represented as a vector-matrix equation, the desired variables are united into vector-original. The applicability of the method is limited by the type of the vector-original being restored: the main part of its components should be equal to the known background value, and a limited number of components should exceed this value. When processing images, this condition means that we have a limited number of bright objects on a constant background.

The application of the proposed method is been demonstrated on the examples of restoration of the blurry and noised images. The task is converted to SLAE, in which the vector-image is a row-column scan of the distorted image, and the being reconstructed vector-original is the row-column scan of the original undistorted image. Computer simulation have been performed in the MATLAB medium, which made it possible to determine the optimal parameters of the stochastic matrixiterative method and to demonstrate its advantages under conditions of additive noise. 
Such conditions are the small image fullness with objects and a large fullness with background values. The influence of additive noise and a priori uncertainty were not considered.

Key words: stochastic matrix-iterative method, matrix-iterative method, SLAE, additive noise, image restoration, point spread function

\section{ВведЕНИЕ}

Многие физические задачи приводятся к задаче решения систем линейных алгебраических уравнений (СЛАУ). К таким задачам относятся, например, обработка изображений $[1,2]$ и обработка сигналов в задачах многоканальной томографии [3].

В векторно-матричной форме СЛАУ имеет вид:

$\mathbf{g}=\mathbf{W f}$,

где $\mathbf{f}$ и $\mathbf{g}$ - векторы, $\mathbf{W}$ - матрица, в общем случае прямоугольная.

Ориентируясь на задачи многоканальной томографии, будем называть $\mathbf{f}$ вектором-оригиналом, $\mathbf{g}$ - вектором-отображением, W-матрицей отображения. В зависимости от размера матрицы отображения СЛАУ (1) может быть недоопределенной или переопределенной. Практические задачи часто приводятся к недоопределенным СЛАУ. Такие системы рассматриваются в данной работе.

Недоопределенная СЛАУ может либо не иметь решения, либо иметь бесчисленное множество решений в случае ее совместности. Условие совместности имеет вид [4]:

$$
\mathbf{W W} \mathbf{W}^{+} \mathbf{g}=\mathbf{g}
$$

где индекс + обозначает псевдообращение матрицы.

При выполнении этого условия существует бесчисленное множество решений, определяемых выражением

$$
\mathbf{f}=\mathbf{W}^{+} \mathbf{g}+\left(\mathbf{I}-\mathbf{W}^{+} \mathbf{W}\right) \mathbf{z}
$$

где $\mathbf{I}$ - единичная матрица, $\mathbf{Z}$ - произвольный вектор $(\operatorname{dim} \mathbf{z}=\operatorname{dim} \mathbf{f})$.

Среди бесчисленного множества решений существует единственное решение с минимальной нормой, которое определяется методом псевдообращения:

$$
\widehat{\mathbf{f}}=\mathbf{W}^{+} \mathbf{g} \text {. }
$$

Если условие (2) совместности СЛАУ не выполняется, то точного решения не существует. В этом случае говорят о приближенном решении [5], которое также находится методом псевдообращения:

$$
\tilde{\mathbf{f}}=\mathbf{W}^{+} \mathbf{g} \text {. }
$$

Это решение является наилучшим в смысле минимума квадратического отклонения $\|\mathbf{W} \tilde{\mathbf{f}}-\mathbf{g}\|^{2}$.

Сравнив (4) и (5), можно заключить, что метод псевдообращения позволяет найти либо точное решение с минимальной нормой, либо наилучшее приближенное решение.

\section{МАТРИЧНО-ИТЕРАЦИОННЫЙ МЕТОД}

Остановимся на неоднозначности решения (3) соВместной СЛАУ. Обычно ищут решение с минимальной нормой методом псевдообращения (4). Однако такое решение не всегда отвечает характеру оцениваемых пространственных или временных распределений, представленных вектором-оригиналом. Так, при обработке изображений метод псевдообращения восстанавливает наиболее равномерное изображение-оригинал, как бы сглаживает его. Такой результат может оказаться весьма далеким от действительного оригинала, например, в случае малоразмерных ярких объектов на протяженном фоне. В этом случае истинным будет другое решение из бесчисленного множества, решение, не обладающее минимальной нормой. Вопрос в том, как его найти.

Известны, например, дескриптивные методы решения СЛАУ [6, 7], которые используют априорную информацию об оригинале. Для их реализации необходимо математически описать имеющуюся априорную информацию об оригинале, дополнить имеющуюся СЛАУ и решать уже эту расширенную систему.

Автором был разработан матрично-итерационный метод, который не предусматривает расширения исходной СЛАУ и, вместе с тем, позволяет находить решение не с минимальной нормой, а в наибольшей степени отвечающее действительному оригиналу. Метод был разработан для восстановления оригинала, включающего незначительное число выбросов на протяженном и известном фоне. Этот метод является вычислительным; подробно он изложен в [8].

Принцип реализации метода следующий. Предположим, что недоопределенная СЛАУ - совместная, имеющая бесчисленное множество решений. Найдем решение методом псевдообращения. Это будет решение с минимальной нормой, то есть сглаженное. В таком решении значения превышающих фон компонент получатся меньше истинных и появятся ложные превышения фона между действительными выбросами. Результат псевдообращения носит колебательный характер, поэтому в полученой оценке оригинала будут присутствовать значения, меньшие фоновых, то есть такие, которых оригинал в действительности не содержит. Эти значения заменяем на фоновые, после чего исключаем из матрицы отображения $\mathbf{W}$ столбцы, составляющие веса замененных компонент, и соответствующим образом корректируем вектор-отображение. В результате СЛАУ станет менее недоопределенной: число столбцов W и компонент вектора-оригинала уменьшается, а размер вектора-отображения остается неизменным. При достаточно малом числе выбросов, превышающих фон, СЛАУ в ходе итераций становится переопределенной. Описанная процедура повторяется до тех пор, пока в очередном решении методом псевдообращения все 
компоненты восстановленного оригинала не окажутся большими либо равными значениям фона.

Задача, однако, усложняется, если система отображения вносит аддитивные шумы, которые, по сути, являются ошибками измерений. В этом случае СЛАУ (1) приобретает вид:

$$
\mathbf{g}=\mathbf{W} \mathbf{f}+\mathbf{n},
$$

где $\mathbf{n}$ - ошибки измерений, которые далее будем называть шумом отображения.

Шум отображения оказывает существенное влияние на решение СЛАУ методом псевдообращения, значительно ухудшая результат или делая восстановление оригинала невозможным. Поэтому при известных вероятностных характеристиках оригинала и шума применяют другие методы, например метод винеровского оценивания [4].

В данной работе предлагается новый, стохастический матрично-итерационный метод, который позволяет получить приемлемое восстановление оригинала по отображению (6) и при этом не требует знания вероятностных характеристик оригинала и не является критичным к знанию вероятностных характеристик шума отображения.

\section{СТОХАСТИЧЕСКИЙ МАТРИЧНО-ИТЕРАЦИОННЫЙ МЕТОД}

Ранее разработанный матрично-итерационный метод приводит к хорошему результату, если на каждой итерации принимаются за фоновые те компоненты восстановленного вектора-оригинала, которые в действительности таковыми и являются. В отсутствие шумов такой ход итераций весьма вероятен. Однако при наличии шума отображения уже на первой итерации за фоновые могут оказаться принятыми компоненты оригинала, значения которых в действительности превышают фон. Такая ситуация возникает, если под воздействием шума эти компоненты восстановились со значениями, меньшими уровня фона. В результате не только превышающая фон компонента будет ошибочно принята за фон, но и внесется ошибка в вектор-отображение $\mathbf{g}$, неправильно будет преобразована матрица отображения $\mathbf{W}$. Это скажется на последующих итерациях и приведет к неизбежным искажениям восстановленного оригинала.

И хотя в целом матрично-итерационный метод восстанавливает оригинал гораздо лучше, чем метод псевдообращения, тем не менее неприятной остается неизбежность и в определенном смысле детерминированность описанного выше хода восстановления.

Если бы отображение регистрировалось многократно, можно было бы ожидать усреднения шума. Однако мы имеем одну реализацию, одно зашумленное отображение. Поэтому с целью уменьшения влияния описанного выше неправильного хода преобразований применим дополнительную стохастическую обработку. В имеющееся зашумленное отображение внесем дополнительную случайность, которую наложим на имеющееся искаженное и зашумленное отображение.
Для этого используем дополнительный вектор $\mathbf{n}_{\text {доп }}$ со случайными $\delta$-коррелированными компонентами с нулевым математическим ожиданием и средним квадратическим отклонением (СКО) $\sigma_{\text {доп }}$ Проведем восстановление вектора-оригинала $\mathbf{f}$ матрично-итерационным методом для $L$ реализаций вектора $\mathbf{n}_{\text {доп }}$ :

$$
\begin{gathered}
\mathbf{g}_{\text {доп } 1}=\mathbf{g}+\mathbf{n}_{\text {доп } 1}=\mathbf{W} \mathbf{f}+\mathbf{n}+\mathbf{n}_{\text {доп } 1,}, \\
\mathbf{g}_{\text {доп } 2}=\mathbf{g}+\mathbf{n}_{\text {доп } 2}=\mathbf{W} \mathbf{f}+\mathbf{n}+\mathbf{n}_{\text {доп } 2,}, \\
\quad \ldots \quad \ldots \\
\mathbf{g}_{\text {доп } L}=\mathbf{g}+\mathbf{n}_{\text {доп } L}=\mathbf{W f}+\mathbf{n}+\mathbf{n}_{\text {доп } L} \cdot
\end{gathered}
$$

Обозначим восстановленные матрично-итерационным методом оригиналы как $\mathbf{f}_{\text {вос } 1}, \mathbf{f}_{\text {вос } 2}, \ldots, \mathbf{f}_{\text {вос } L}$. Окончательное решение получим усреднением этих $L$ результатов:

$$
\widehat{\mathbf{f}}_{\mathrm{cT}}=\frac{\sum_{i=1}^{L} \mathbf{f}_{\mathrm{Boc} i}}{L} .
$$

Формулы (7) и (8) описывают стохастический матрично-итерационный метод. Далее рассматривается его применение в задаче восстановления изображенияоригинала по размытому и зашумленному отображению.

\section{ПОСТАНОВКА ЗАДАЧИ}

Обозначим размеры размытого и зашумленного дискретизированного отображения как $M \times M$ элементов разрешения. Это то изображение, которое регистрируется и по которому восстанавливается изображение-оригинал. Размытие оригинала происходит за счет свертки его с известной функцией рассеяния точки (ФРТ). Размеры ФРТ составляют $K \times K$ элементов разрешения. При этом число элементов разрешения в восстанавливаемом оригинале будет $N \times N$, где $N=M+K-1$ при нечетном $K$.

На размытое изображение накладывается аддитивный белый гауссовский шум с нулевым средним и СКО $\sigma_{n}$.

Ставится задача по размытому и зашумленному отображению восстановить оригинал.

\section{РЕЗУЛЬТАТЫ КОМПЬЮТЕРНОГО МОДЕЛИРОВАНИЯ}

Решать поставленную задачу будем с использованием компьютерного моделирования в среде MATLAB. Цель моделирования - реализовать стохастический матрично-итерационный метод восстановления изображения-оригинала по его размытому и зашумленному отображению согласно алгоритму (7), (8) и продемонстрировать преимущества этого метода перед методом псевдообращения и матрично-итерационным методом.

В экспериментах отображение имеет размер $7 \times 7 \mathrm{~cm}^{2}$. ФРТ имеет гауссовскую форму и ограничена 
областью $2,5 \times 2,5 \mathrm{~cm}^{2}$. Элемент разрешения составляет 0,5 0,5 $\mathrm{cm}^{2}$. Таким образом, матрица дискретизированного отображения имеет размер $M \times M=14 \times 14$, а матрица дискретизированной ФРТ $-K \times K=5 \times 5$. Матрица восстанавливаемого оригинала будет при этом иметь размер $N \times N=18 \times 18$. Аддитивный шум отображения $\mathbf{n}-$ белый гауссовский с СКО $\sigma_{n}$, которое в экспериментах меняет значение.

Преобразование оригинала в отображение описывается уравнением (6) в котором $\mathbf{g}$ и $\mathbf{f}$ представляют собой строчно-столбцовые развертки соответствующих изображений; при этом $\operatorname{dim} \mathbf{g}=196 \times 1, \operatorname{dim} \mathbf{f}=324 \times 1$, $\operatorname{dim} \mathbf{W}=196 \times 324, \operatorname{dim} \mathbf{n}=196 \times 1$.

Исходное изображение-оригинал, размытый оригинал без шума и окончательное зашумленное отображение с $\sigma_{n}=0,05$ и $\sigma_{n}=0,1$ показаны на рисунке 1. Фон оригинала постоянный и составляет 3 (в единицах интенсивности).

На рисунке 2 показаны результаты восстановления оригинала по отображению, показанному на рисунке 1г.

Рисунок 2 позволяет качественно сравнить результаты восстановления оригинала тремя методами - псевдообращения, матрично-итерационным и стохастическим матрично-итерационным. Для количественной оценки качества восстановления будем использовать квадратическую ошибку восстановления $\delta=(\widehat{\mathbf{f}}-\mathbf{f})^{T}(\widehat{\mathbf{f}}-\mathbf{f})$.

Восстановление методом псевдообращения выполнено с улучшающей постобработкой, в ходе которой все значения восстановленного оригинала, меньшие фонового значения, были приняты равными интенсивности фона. В стохастическом матрично-итерационном методе число реализаций дополнительного случайного вектора $\mathbf{n}_{\text {доп }}$ принято $L=30$.

Как видно из рисунка 2, метод псевдообращения не позволяет восстановить оригинал, квадратическая ошибка восстановления составляет $\delta_{\text {ПО }}=63,9456$. Матрично-итерационный метод дает представление об оригинале, его квадратическая ошибка $\delta_{\mathrm{MИ}}=3,6360$. Стохастический матрич-

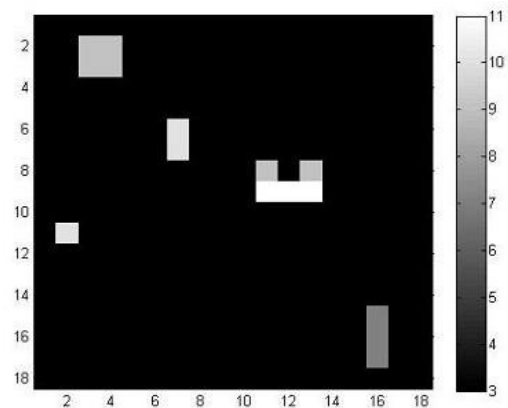

a

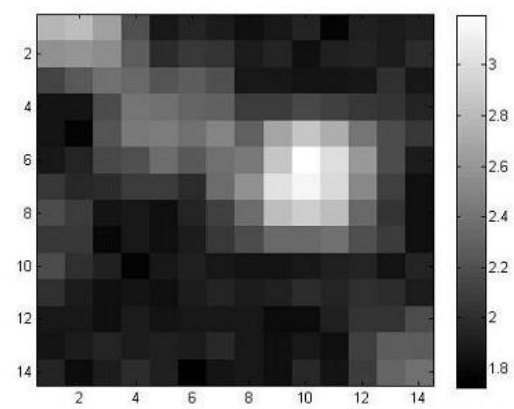

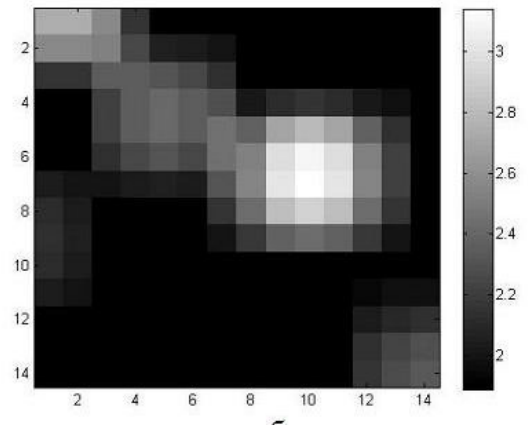

6

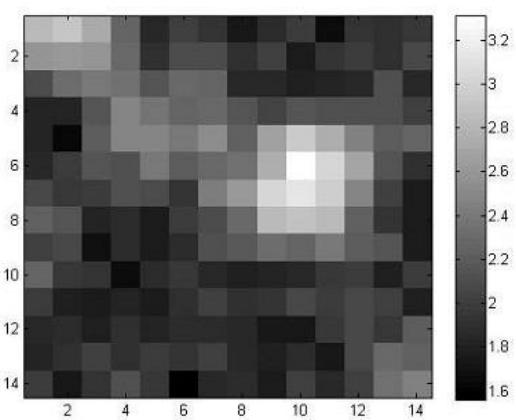

Рис. 1. Формирование отображения: оригинал (а), размытый оригинал (б), отображение с СКО шума $\sigma_{n}=0,05$ (в) и $\sigma_{n}=0,1$ (г)
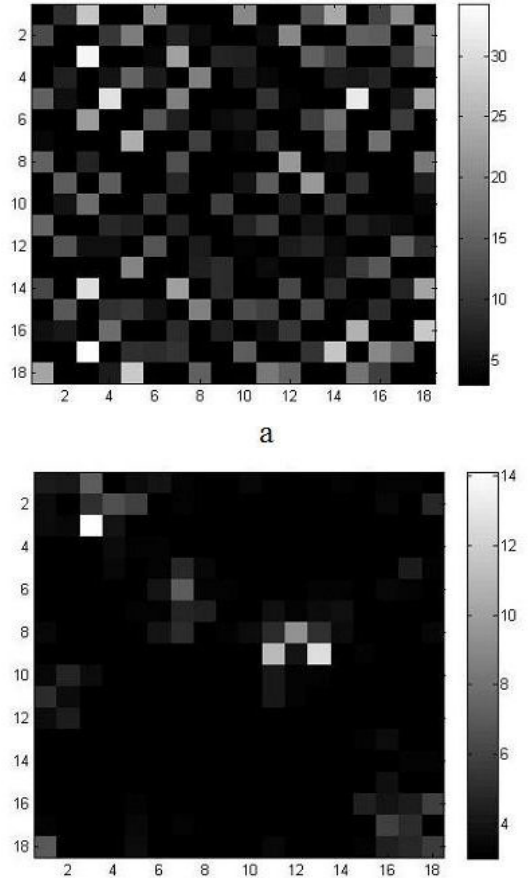

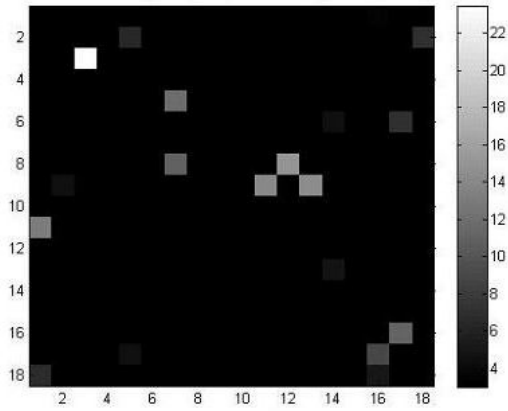

6

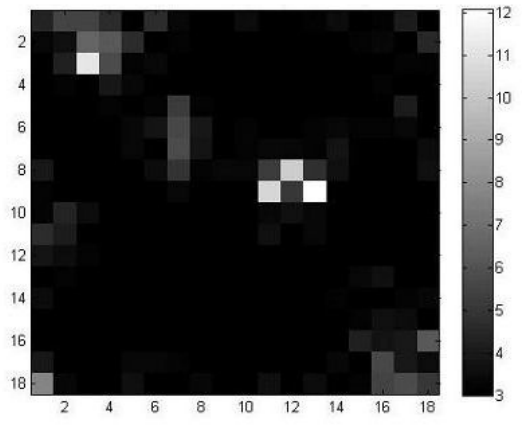

Рис. 2. Восстановленный оригинал: методом псевдообращения (а), матрично-итерационным методом (б), стохастическим матрично-итерационным методом с СКО компонент дополнительного случайного вектора $\sigma_{\text {доп }}=0,1$ (в) и $\sigma_{\text {доп }}=0,15$ (г) 


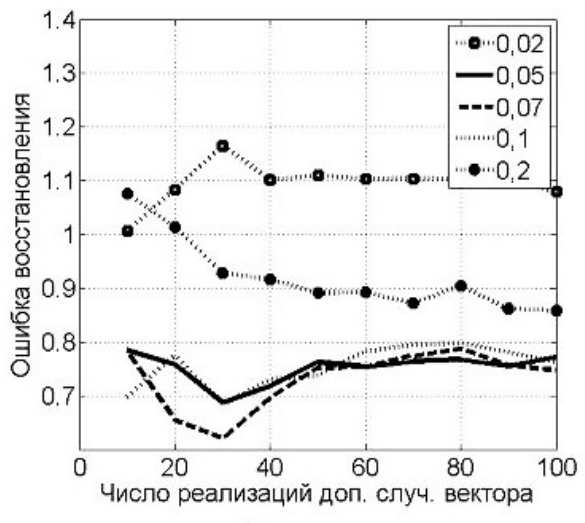

a

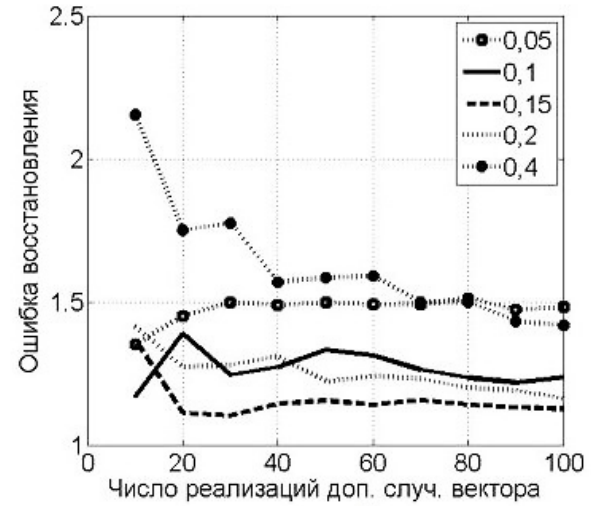

6

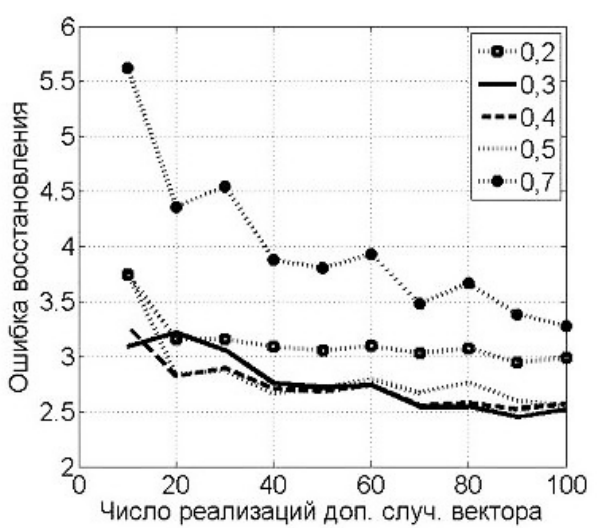

B

Рис. 3. Влияние числа реализаций дополнительного случайного вектора на качество восстановления оригинала стохастическим матрично-итерационным методом при СКО шума отображения $\sigma_{n}=0,05\left(\right.$ а), $\sigma_{n}=0,1$ (б) и $\sigma_{n}=0,3$ (в); в углах графиков обозначены значения $\sigma_{\text {доп' }}$ соответствующие каждой зависимости

но-итерационный метод дает более качественный результат: при $\sigma_{\text {доп }}=0,1$ получается $\delta_{\mathrm{CMИ}}=1,2449$, а при $\sigma_{\text {доп }}=0,15-\delta_{\text {СМИ }}=1,1020$.

Рисунки 3 и 4 иллюстрируют влияние параметров стохастического матрично-итерационного метода на результаты восстановления оригинала. Такими параметрами являются число реализаций $L$ дополнительного случайного вектора и СКО компонент этого вектора $\sigma_{\text {доп }}$

На рисунке 3 показаны зависимости ошибок восстановления оригинала от числа реализаций $L$ дополнительного случайного вектора. Эксперименты проведены при трех значениях СКО шума отображения $\sigma_{n^{\prime}}$ равных 0,05, 0,1 и 0,3. СКО компонент дополнительного случайного вектора $\sigma_{\text {доп }}$ соответствующие всем зависимостям, показаны в углах графиков. Сплошной линией показаны зависимости, полученные (и все-таки мне кажется, что Word ошибается, везде требуя двойное «н»!) при значении $\sigma_{\text {доп }}=\sigma_{n}$;

Как видно из графиков на рисунке 3, зависимость от числа реализаций связана с СКО компонент дополнительного случайного вектора $\sigma_{\text {доп }}$ При значениях $\sigma_{\text {доп' }}$ близких к СКО шума отображения $\sigma_{n}$, такая зависимость незначительна, а при $\sigma_{\text {доп }}>>\sigma_{n}$ ошибка восстановления заметно уменьшается с увеличением $L$, оставаясь при этом сравнительно большой.

На рисунке 4 показана зависимость ошибки восстановления оригинала от относительного СКО компонент дополнительного случайного вектора $\sigma_{\text {доп } / \sigma_{n}}$.

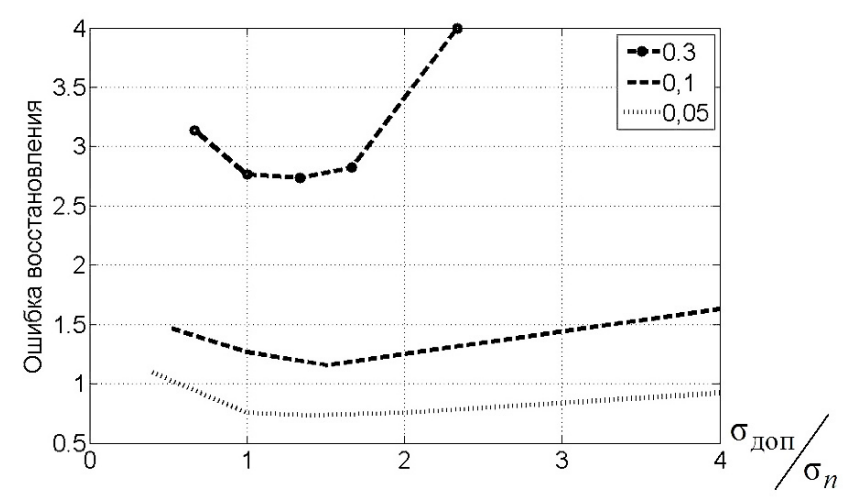

Рис. 4. Влияние СКО компонент дополнительного случайного вектора на ошибку восстановления 
Единица на оси абсцисс соответствует равенству значений СКО дополнительного случайного вектора и СКО шума отображения: $\sigma_{\text {доп }}=\sigma_{n}$. Цифры в углу графика это значения СКО шума отображения для каждой зависимости. По оси ординат отложены ошибки восстановления, усредненные по числу реализаций дополнительного случайного вектора.

Из графиков на рисунке 4 видно, что существует оптимальное значение СКО компонент дополнительного случайного вектора $\sigma_{\text {доп' }}$ при котором получается минимальная ошибка восстановления. При всех трех рассмотренных шумах отображения это оптимальное значение равно или незначительно превышает СКО шума отображения $\sigma_{n}$.

Целью следующих экспериментов является сравнительный анализ качества восстановления оригинала матрично-итерационным и стохастическим матрично-итерационным методами. Эксперименты проводились и с методом псевдообращения, однако все они показали неработоспособность этого метода в условиях моделировавшихся шумов отображения: ошибка восстановления методом псевдообращения на порядок выше, чем матрично-итерационным и стохастическим матрично-итерационным методами.

На рисунке 5 приведены зависимости ошибок восстановления оригинала матрично-итерационным и стохастическим матрично-итерационным методами от СКО шума отображения.

В каждом эксперименте проведено усреднение по 20 реализациям шума отображения с одним и тем же СКО. В стохастическом матрично-итерационном методе число реализаций дополнительного случайного вектора $L=10$, а СКО его компонент $\sigma_{\text {доп }}=\sigma_{n}$.

Как видно из графиков на рисунке 5 , стохастический матрично-итерационный метод имеет преимущество по точности восстановления перед матрично-итерационным методом при всех исследованных значениях

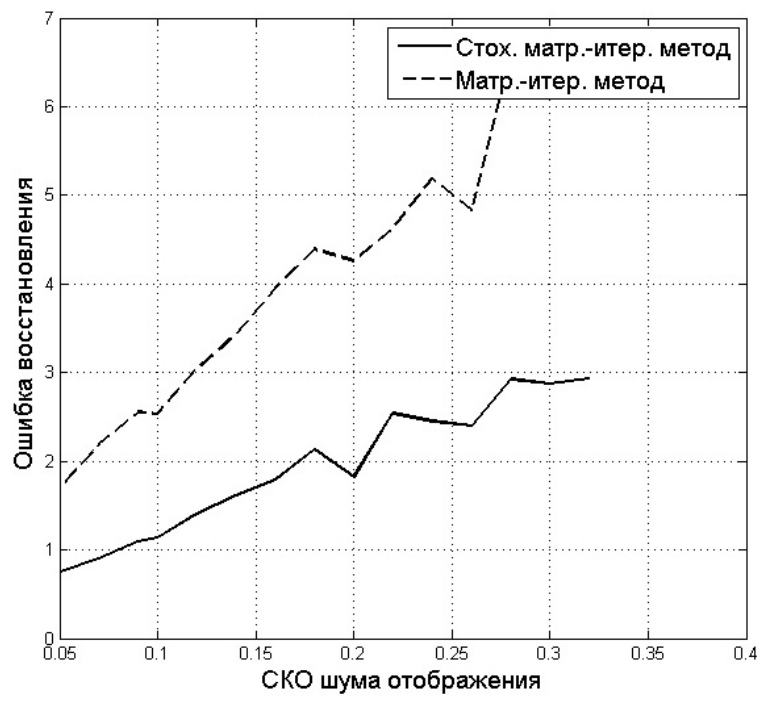

Рис. 5. Влияние шума отображения на качество восстановления оригинала матрично-итерационным и стохастическим матрично-итерационным методами
СКО шума отображения, причем преимущество это увеличивается с увеличением дисперсии шума.

\section{ЗАКЛЮЧЕНИЕ}

Предложенный в работе новый, стохастический матрично-итерационный метод (вторую зпт. убираю, а первая несет смысловую нагрузку: не новый стохастический..., а новый, который называется «стохастический ...») был исследован с помощью компьютерных экспериментов. Эксперименты показали, что в условиях аддитивных шумов отображения метод псевдообращения не работает, а матрично-итерационный и стохастический матрично-итерационный методы позволяют существенно улучшить отображение и получить представление об оригинале.

Исследовалась зависимость точности восстановления оригинала от параметров стохастического матрично-итерационного метода: от числа реализаций $L$ дополнительного случайного вектора и от СКО компонент этого вектора $\sigma_{\text {доп. }}$ Результаты показали, что оптимальным является значение $\sigma_{\text {доп, }}$ равное или незначительно превышающее СКО шума отображения. Что касается числа реализаций дополнительного случайного вектора, то достаточным можно считать 10 его реализаций; увеличение этого числа незначительно уменьшает ошибку восстановления при оптимальном значении $\sigma_{\text {доп }}$

Исследовалась зависимость ошибок восстановления оригинала от СКО шума отображения методом псевдообращения, матрично-итерационным и стохастическим матрично-итерационным методами. Метод псевдообращения не позволил восстановить оригинал ни при одном из значений СКО шума из моделировавшихся в экспериментах: его ошибка восстановления оказалась на порядок больше, чем в матрично-итерационном и стохастическом матрично-итерационном методах. Из двух последних методов очевидное преимущество по качеству восстановления показал стохастический матрично-итерационный метод при всех рассматривавшихся условиях экспериментов.

\section{СПИСОК ЛИТЕРАТУРЫ}

1. Прэтт У. Цифровая обработка изображений. В 2-х кн. : пер. с англ. - М. : Мир, 1982. - 790 с.

2. Обработка изображений и цифровая фильтрация / под ред. Т. Хуанга. - М. : Мир, 1979. - 318 с.

3. Самойленко М.В. Обработка сигналов в задачах локационных измерений и оценивания. - М. : Изд. дом «Спектр», 2016. - 260 с.

4. Самойленко В.И., Пузырев В.А., Грубрин И.В. Техническая кибернетика : учеб. пособие. - М. : Изд-во МАИ, 1994. - 280 с.

5. Гантмахер Ф.Р. Теория матриц. - 4-е изд. - М. : Наука, 1988. - 552 с.

6. Воскобойников Ю.Е., Мицель А.А. Современные проблемы прикладной математики. Ч. І. Лекционный курс : учеб. пособие. / Томский гос. университет си- 
стем управления и радиоэлектроники (ТУСУР). - Томск, 2015. -136 c.

7. Кирьянов Д.В., Кирьянова Е.Н. Вычислительная физика. - М. : Полибук Мультимедиа, 2006. - 352 с.

8. Самойленко М.В. Матрично-итерационный метод решения системы линейных уравнений и его применение в томографическом сканировании пространства с использованием радиолокационной станции // Научно-технический вестник информационных технологий, механики и оптики. - 2018. - Т. 18. № 3. - С. 437-446.

\section{REFERENCES}

1. Prett U. Tsifrovaia obrabotka izobrazhenii. V 2-kh kn. per. s angl. [Digital Picture Processing. Translated from Engl.]. Moscow, Mir Publ., 1982.

2. Obrabotka izobrazhenii i tsifrovaia filtratsiia. Pod red. T. Huanga, per. s angl. [Picture Processing and Digital Filtering. Edited by T. Huang. Translated from Engl.]. Moscow, Mir Publ., 1979. 318 p.

3. Samoilenko M.V. Obrabotka signalov v zadachakh lokatsionnykh izmerenii i otsenivaniia [Signal Processing for Tasks on Location Measurements and Estimation]. Moscow, Spektr Publ., 2016. 260 p.

4. Samoilenko V.I., Puzyrev V.A., Grubrin I.V. Tekhnicheskaia kibernetika. Ucheb. posobie [Engineering Cybernetics. Textbook]. Moscow, MAI Publ., 1994. 280 p.
5. Gantmakher F.R. Teoriia matrits. 4-e izd. [Matrix Theory. The 4th Edition]. Moscow, Nauka Publ., 1988. $552 \mathrm{p}$.

6. Voskoboinikov lu.E., Mitsel A.A. Sovremennye problemy prikladnoi matematiki. Ch. I. Lektsionnyi kurs. Ucheb. posobie [Modern Problems of the Applied Mathematics. Part 1. Lectures. Textbook]. Tomsk, Tomsk State University of Control Systems and Radioelectronics (TUSUR) Publ., 2015. 136 p.

7. Kirianov D.V., Kirianova E.N. Vychislitelnaia fizika [Computer Physics]. Moscow, Polibuk Multimedia Publ., 2006. 352 p.

8. Samoilenko M.V. Matrichno-iteratsionnyi metod resheniia sistemy lineinykh uravneni i ego primenenie v tomograficheskom skanirovanii prostranstva s ispolzovaniem radiolokatsionnoi stantsii [Matrix-Iterative Solution Method for System of Linear Equations and Its Application in Space Tomography Scanning Using Radar]. Nauchno-tekhnicheskii vestnik informatsionnykh tekhnologii, mekhaniki i optiki [Scientific and Technical Journal of Information Technologies, Mechanics and Optics], 2018, vol. 18, no. 3, pp. 437-446. 